\title{
High resolution interferometry of the QSO $1422+202$
}

\author{
L.B. Bååth ${ }^{1}$, F. Mantovani ${ }^{2}$, and F.T. Rantakyrö ${ }^{2}$ \\ 1 Centre for Imaging Technologies, Halmstad University, S-301 18 Halmstad, Sweden \\ ${ }^{2}$ Istituto di Radioastronomia del C.N.R., Via P. Gobetti, I-40129 Bologna, Italy
}

Received November 10, 1996; accepted January 29, 1997

\begin{abstract}
We present VLA A-array observations at 8.4 and $15 \mathrm{GHz}$ and European VLBI Network (EVN) observations at $1.6 \mathrm{GHz}$ of the radio source $1422+202$. It is suggested that $1422+202$ is a Medium-size Object in the evolutionary sequence from Compact Steep-spectrum Sources to larger sized radio sources. The VLBI data were analysed with the phase referencing technique and we show that the EVN can work as a phase stable instrument for separations between the calibrator source and the target source up to $\sim 10^{\circ}$. With the VLA and VLBI observations we investigate some of the issues about the nucleus of $1422+202$ and we discuss the possible cause for the low frequency variability detected while monitoring the source.
\end{abstract}

Key words: galaxies: jets — quasars: QSO 1422+202 radio continuum: galaxies

\section{Introduction}

The radio source $1422+202(4 \mathrm{C} 20.33)$ is a steep spectrum radio source identified with a quasar of redshift 0.871 and visual magnitude 17.65 (Véron \& Véron 1987). It was recognized as a member of the Compact Steep Spectrum class of sources by Mantovani et al. (1992) in their investigation of the arcsecond scale structure of a sample of 19 steep spectrum sources showing variability at low frequency. Reported as a variable source at $408 \mathrm{MHz}$ by Fanti et al. (1983), 1422+202 did not change in flux density with time at higher frequencies (Padrielli et al. 1987) for several years. In the VLA image at $5 \mathrm{GHz}$ (Mantovani et al. 1992) 1422+202 exhibits a structure elongated north-south, with a faint region of emission offaxis close to the southern bright hot spot.

We present here new VLA A-array observations at 8.4 and $15 \mathrm{GHz}$ and VLBI observations at $1.6 \mathrm{GHz}$ made with the European VLBI Network (EVN). The VLBI observations were designed to apply directly the wide

Send offprint requests to: F. Mantovani field mapping technique to the data set from the correlator. However, due to technical problems during the data recording at some stations we choose to analyse the data by applying the phase referencing technique. The main aim was the detection of both the core and the southern hot spot which lies $\sim 8^{\prime \prime}$ away, as suggested by beating in the fringe visibility on the baseline Effelsberg-Westerbork obtained in a previous EVN pilot experiment at $1.6 \mathrm{GHz}$. The absolute positions, relative to the calibration source OQ208, obtained for the detected components in the VLBI field of view, were then compared with those achieved in the VLA image at $15 \mathrm{GHz}$.

Combining the VLA and VLBI observations we investigated some of the issues regarding the nucleus of $1422+202$. Before these observations it was not clear where this source fits in the CSSs scheme, which were the physical processes causing the helical structure shown by the jet, the nature of the asymmetrical structure and the cause of the variability at low frequency.

\section{VLA and VLBI observations}

\subsection{VLA observations}

The source was observed with the VLA (Thompson et al. 1980) in the A configuration on 1990 May 17 at 8.4 and $15 \mathrm{GHz}$ (resolution $\sim 0.30^{\prime \prime}$ and $\sim 0.17^{\prime \prime}$ respectively) for about 15 minutes. Two IF channels each with a $50 \mathrm{MHz}$ bandwidth and separated by $50 \mathrm{MHz}$ were used at both frequencies. The data were calibrated using the standard VLA calibrators and the source imaged with the NRAO AIPS programs.

\subsection{The arcsecond scale structure}

The VLA image of $1422+202$ at $8.4 \mathrm{GHz}$ shows that the source structure is mainly elongated north-south. It contains several blobs of emission labelled from $a$ to $e$ in Fig. 1. A faint extended region of emission (component $f$ ) is also seen off-axis near component $e$. This last component and component $d$ are not detected at $15 \mathrm{GHz}$ (see Fig. 2 ). Most of the emission from the remaining components 
is resolved out. Component $e$ shows a ridge of emission along the major axis and the bright peak appears resolved in two components. Component $b$, unresolved at $8.4 \mathrm{GHz}$ shows here an extension in Position Angle (PA) $\sim 40^{\circ}$. The brightness distribution can be fitted with a two Gaussian model. If the bright peak of emission in $b$ is instead fitted with a single circular Gaussian model and then subtracted, the residual map shows $1-2 \mathrm{mJy}$ left south-west of the peak. So we believe that the extension is real.

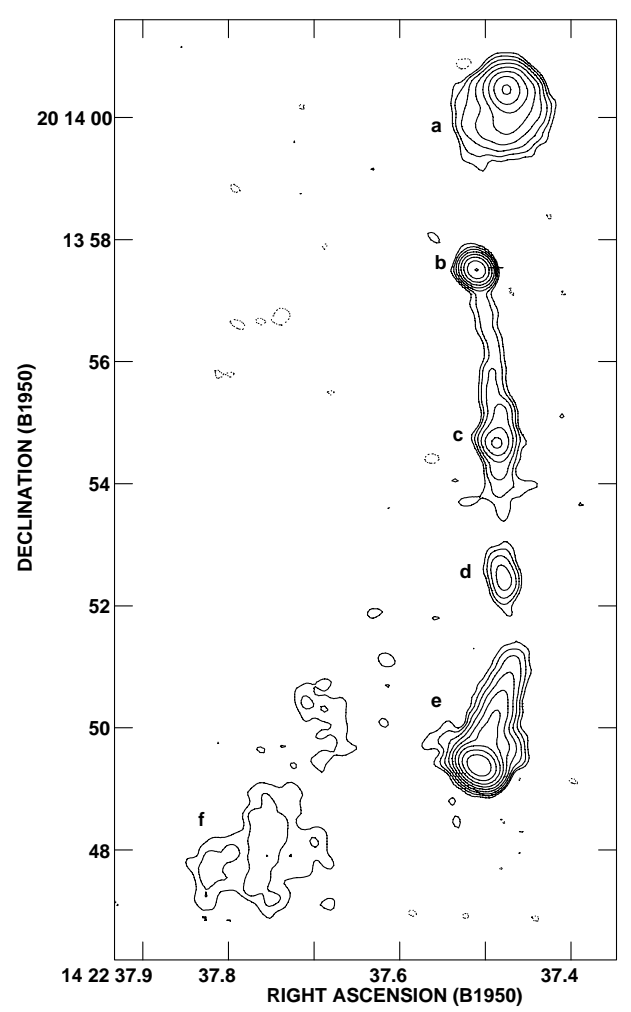

Fig. 1. $8.4 \mathrm{GHz}$ VLA. The beam is $0.30^{\prime \prime} \times 0.27^{\prime \prime}$ in PA $50^{\circ}$. The noise is $0.06 \mathrm{mJy}^{\text {beam }}{ }^{-1}$. Contours are at $-0.2,0.2,0.4$, $0.6,0.8,1,2,4,8,16,32,64,128,256$ mJy beam $^{-1}$. The peak flux density is $102.1 \mathrm{mJy}^{\text {beam }^{-1}}$. Component $b$ is believed to be the core

Previous observations of $1422+202$ made with MERLIN at $408 \mathrm{MHz}$ and with the VLA at $5 \mathrm{GHz}$ can be found in Mantovani et al. (1992). The three VLA images of $1422+202$ at $5,8.4$ and $15 \mathrm{GHz}$ were convolved with the same circular Gaussian beam (FWHM 0.5"). The spectral index distributions were obtained for two ranges, $5 \mathrm{GHz}-8.4 \mathrm{GHz}$ and $8.4 \mathrm{GHz}-15 \mathrm{GHz}$. The spectral index is everywhere much steeper than $0.4\left(S \propto \nu^{-\alpha}\right)$. Some flattening of the slope is visible only for component $b$. We can say more about the spectral shape of the emission in $b$ taking into account the MERLIN map at $408 \mathrm{MHz}$.

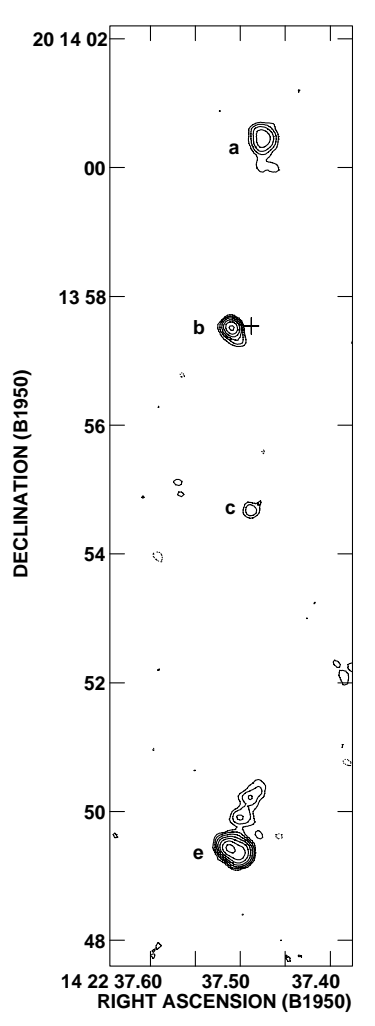

Fig. 2. $15 \mathrm{GHz}$ VLA map. The beam is $0.17^{\prime \prime} \times 0.15^{\prime \prime}$ in PA $50^{\circ}$. The noise is $0.1 \mathrm{mJy}^{\text {beam }}{ }^{-1}$. Contours are at $-0.4,0.4,0.6$, $0.8,1,2,4,8,16,32,64$ mJy beam $^{-1}$. The peak flux density is $34.0 \mathrm{mJy}^{\text {beam }^{-1}}$. A cross marks the position of the optical counterpart

There the component $b$ was not detected, and we can put an upper limit of $\sim 5 \mathrm{mJy}$ to its emission. From the VLA convolved maps at $5,8.4$ and $15 \mathrm{~Hz}$ we have 34,26 and $15 \mathrm{mJy}$ peak respectively, suggesting that $1422+202$ has a Giga-Hz-peaked Spectrum core which peaks about $3-5 \mathrm{GHz}$. This is confirmed if we also plot the flux density of $15.1 \mathrm{mJy}$ we got at $1.6 \mathrm{GHz}$ from the VLBI map (see Table 1). Such a value fits with a curved spectral index peaking at $\sim 4 \mathrm{GHz}$. The component $b$ is believed to be the core of $1422+202$.

The overall structure of the source is thus rather asymmetric, with a long collimated wiggling jet pointing south, no evidence at the detection limit of our maps of a counterjet, a weak nearby hot spot to north (component $a$ ) and a bright hot spot at the end of the jet on the opposite side (component $e$ ). The jet major axis changes in PA several time along its path. The core, for example shows an extension in $\mathrm{PA} \sim 40^{\circ}$, quite different from the $\mathrm{PA}$ of the ridge of emission in component $e$ which is $\sim-25^{\circ}$. Thus we suggest that the jet is the projected image of a helical precessing jet. 


\subsection{VLBI observations}

The VLBI observations were made at $1.6 \mathrm{GHz}$ on 1987 March 1 with the EVN recording with the MarkIIIA terminal in Mode B and standard setup. The source $1422+202$ was tracked for about 11 hours together with the calibration source OQ 208, observed for three scans, 13 min long each, regularly spaced over the experiment. The data recorded at each station were correlated at the MarkIII correlator of the Max-Planck-Institut für Radioastronomie.

The raw data output from the correlator, were read with the MK3IN-program (Bååth \& Mantovani 1991) and analysed with AIPS. Our aim was the detection and the imaging of the two components (the core and the south hot spot) separated by $\sim 8^{\prime \prime}$ detected during a VLBI pilot experiment with the short baseline Effelsberg-Westerbork. The wide field mapping technique described in Bååth (1991) could not be used directly for finding fringes. This technique requires a phase-cal signal in each independent IF-channel to allow the removal of the phase differences between the IF-channels in the postprocessing stage. Unfortunately, the phase-cal signal was not injected at all stations so we had to follows a different strategy. The fringes were searched with the task CALIB for each IF channel independently on the calibrator source OQ 208. The solutions found for OQ 208 were applied to $1422+202$, which is $9.6^{\circ}$ away. This technique is equivalent to using a phase-cal signal, and allowed us to thereafter remove the single and multiband band delays on $1422+202$. The multiband delays were fitted after averaging each IF over the frequency channels. In other words, the phase referencing technique, which usually requires to observe switching between the calibrator and the target source with a short duty cicle, was successfully applied even in this case where the calibration source was observed only three times.

The source $1422+202$ was then imaged without obtaining any further fringe solution. It showed up with an absolute position which agrees with the VLA position. This will be discussed further in Sect. 3.2. The image had a well defined compact component coinciding with the expected position of the core. We then proceeded by fitting for station based phase offsets in order to further focus the image.

\subsection{The milliarcsecond scale structure}

The VLBI map is shown in Fig. 3. The map was obtained by restoring the field with a coarse beam of $0.15^{\prime \prime} \times 0.15^{\prime \prime}$. All the extended structure has been resolved out. Only two components were detected in the imaged field, almost aligned north-south, separated by $\sim 8^{\prime \prime}$. The main component lies in the area were the core of the source is. The second component is weaker and slightly extended. Its position coincides with that of the south hot spot seen in the VLA maps.

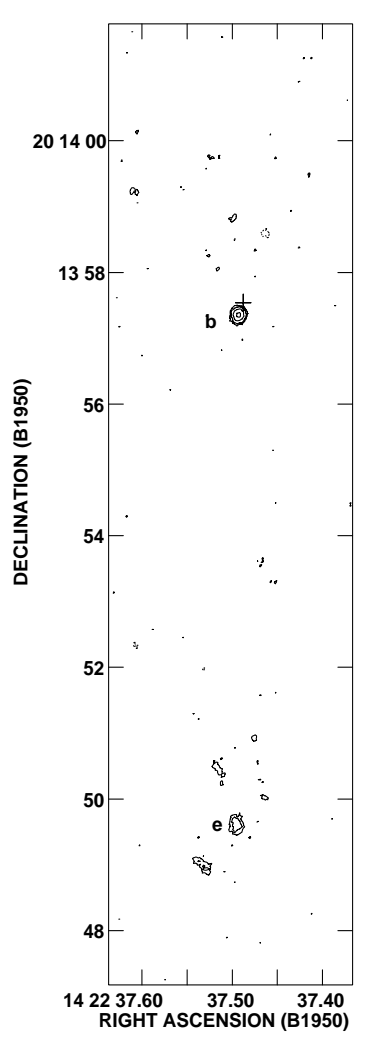

Fig. 3. $1.6 \mathrm{GHz}$ VLBI map. The beam is $0.15^{\prime \prime} \times 0.15^{\prime \prime}$. The noise is $0.5 \mathrm{mJy}^{\text {beam }}{ }^{-1}$. Contours are at $-1.5,1.5,3,5,7$, $9,11,13,15,17,20 \mathrm{mJy}_{\text {beam }^{-1}}$. The peak flux density is $14.2 \mathrm{mJy}$ beam $^{-1}$. A cross marks the position of the optical counterpart

\section{Results}

\subsection{The observational parameters}

The observational parameters of the radio observations are summarized in Table 1.

\subsection{Source position}

The positions we obtained for the VLBI core component of $1422+202$ are referred to those of OQ 208. Before making any comparisons between the VLA and VLBI positions one has to take the following issues into consideration.

- The differences between the positions used as input at the VLBI correlator and the VLA positions for OQ 208 give (VLBI-VLA): $\alpha(\mathrm{B} 1950.0)=0.0133^{\mathrm{s}}$ and $\delta(\mathrm{B} 1950.0)=0.2909^{\prime \prime}$. This difference accounts for both the elliptical aberration $\left(\sim 0.13^{\prime \prime}\right.$ and $\sim 0.18^{\prime \prime}$ in R.A. and Dec. respectively; see for example Aoki et al. 1983) and the difference between the VLBI reference frame and the VLA we derived $\left(<0.05 \pm 0.3^{\prime \prime} \mathrm{rms}\right.$ in B1950.0 coordinates). The elliptical aberration had 
Table 1. Observational parameters of $1422+202 . \sigma_{i}$ is the rms noise in the total intensity map far from the source of the emission. The positions of the components have been estimated from two-dimensional Gaussian fits to the image. The peak flux densities are those at the pixel of maximum brightness and the integrated flux densities have been obtained by specifying small boxes around the components

\begin{tabular}{|c|c|c|c|c|c|c|c|c|c|c|c|c|c|c|c|}
\hline \multirow{2}{*}{$\begin{array}{l}\text { Array } \\
\text { name }\end{array}$} & \multirow[t]{2}{*}{ Date } & \multirow{2}{*}{$\begin{array}{r}\nu \\
(\mathrm{MHz})\end{array}$} & \multicolumn{3}{|c|}{ Beam } & \multirow{2}{*}{$\begin{array}{l}\sigma_{\mathrm{i}} \\
(\mathrm{mJy})\end{array}$} & \multirow[t]{2}{*}{ Comp. } & \multicolumn{3}{|c|}{$\mathrm{RA}(1950)$} & \multicolumn{3}{|c|}{$\overline{\mathrm{DEC}}(1950)$} & \multirow{2}{*}{$\begin{array}{r}S_{\text {peak }} \\
(\mathrm{mJy})\end{array}$} & \multirow{2}{*}{$\begin{array}{r}S_{\text {tot }} \\
(\mathrm{mJy})\end{array}$} \\
\hline & & & $\left({ }^{\prime \prime}\right)$ & $\left({ }^{\prime \prime}\right)$ & $\left(^{\circ}\right)$ & & & (h) & $(\mathrm{m})$ & $(\mathrm{s})$ & $\left({ }^{\circ}\right)$ & $\left({ }^{\prime}\right)$ & $\left({ }^{\prime \prime}\right)$ & & \\
\hline$\overline{\mathrm{VLA}}$ & 90 May 17 & 8415 & 0.30 & 0.26 & 50 & 0.06 & $\bar{a}$ & 14 & 22 & 37.47 & 20 & 14 & 00.44 & 28.6 & 85.6 \\
\hline - & - & - & - & - & - & - & $\mathrm{b}$ & 14 & 22 & 37.51 & 20 & 13 & 57.50 & 26.6 & 26.6 \\
\hline - & - & - & - & - & - & - & $\mathrm{c}$ & 14 & 22 & 37.49 & 20 & 13 & 54.68 & 7.3 & 19.4 \\
\hline - & - & - & - & - & - & - & d & 14 & 22 & 37.48 & 20 & 13 & 52.44 & 2.4 & 4.6 \\
\hline - & - & - & - & - & - & - & $\mathrm{e}$ & 14 & 22 & 37.51 & 20 & 13 & 49.39 & 100.1 & 206.9 \\
\hline - & - & - & - & - & - & - & $\mathrm{f}$ & 14 & 22 & 37.75 & 20 & 13 & 47.90 & 0.8 & 13.6 \\
\hline VLA & 90 May 17 & 14965 & 0.17 & 0.15 & 50 & 0.1 & $\mathrm{a}$ & 14 & 22 & 37.47 & 20 & 13 & 57.45 & 6.2 & 17.4 \\
\hline - & - & - & - & - & - & - & $\mathrm{b}$ & 14 & 22 & 37.51 & 20 & 13 & 57.51 & 15.1 & 18.7 \\
\hline - & - & - & - & - & - & - & c & 14 & 22 & 37.49 & 20 & 13 & 54.68 & 1.7 & 2.3 \\
\hline- & - & - & - & - & - & - & $\mathrm{e}$ & 14 & 22 & 37.51 & 20 & 13 & 49.40 & 31.4 & 92.4 \\
\hline EVN & 87 Mar 01 & 1.666 & 0.15 & 0.15 & - & 0.5 & $\mathrm{~b}$ & 14 & 22 & 37.49 & 20 & 13 & 57.35 & 14.7 & 15.1 \\
\hline- & - & - & - & - & - & - & d & 14 & 22 & 37.50 & 20 & 13 & 49.62 & 5.2 & 7.4 \\
\hline
\end{tabular}

to be corrected for, as source positions in b1950.0 coordinates were used in the correlator, which does not account for elliptical aberration.

- The positional accuracy for a VLA observation is estimated to be better than 0.1" (Perley et al. 1982).

The cross in Figs. 2-3 marks the position of the optical counterpart for $1422+202$ as given by Argue et al. (1973). Table 2 presents the differences between the VLBI, $\mathrm{VLA}_{15 \mathrm{GHz}}$, and optical positions.

Table 2. Positional differences for the core and the south hot spot in $1422+202$

\begin{tabular}{lll}
\hline Regions & $\begin{array}{l}\Delta \alpha \\
{[\mathrm{sec}]}\end{array}$ & $\begin{array}{l}\Delta \delta \\
{\left[{ }^{\prime \prime}\right]}\end{array}$ \\
\hline (VLA-VLBI)core & 0.02 & +0.26 \\
(VLA-VLBI)hot spot & 0.01 & -0.22 \\
VLAcore-Optical & 0.02 & -0.03 \\
\hline
\end{tabular}

We estimate the VLBI positional accuracy of $1422+202$ to be about of the same order of magnitude as the $\mathrm{VLA}_{15 \mathrm{GHz}}$ positional accuracy. This means that the positions derived from the VLBI, $\mathrm{VLA}_{15 \mathrm{GHz}}$, and optical observations do overlap inside the given errors.

\section{Discussion}

\subsection{Source structure}

The inclusion of the quasar $1422+202$ in the CSS class of source by Mantovani et al. (1992) was mainly based on its relatively small linear size of $43 \mathrm{kpc}\left(H_{0}=\right.$
$100 \mathrm{~km} \mathrm{~s}^{-1} \mathrm{Mpc}^{-1}$ ) and on its overall spectral index in the radio band. Since it was clear we were dealing with a compact object the suggestion of $1422+202$ as a candidate CSS was not considered a compelling choice in a period where the understanding of the CSSs class of object was growing. Moreover, the source exhibits a radio power of $\sim 510^{27} \mathrm{~W} / \mathrm{Hz}$ at $178 \mathrm{MHz}$ which is comparable to the radio power found for the CSSs selected from the $3 \mathrm{CR}$ catalogue. On the other hand the linear size of $1422+202$, exceeds the selection criteria (linear size $\leq 15 \mathrm{kpc}$ ) set by Fanti et al. (1995) in a paper in which the concept of a connection between CSSs and larger sized radio sources is introduced: the CSSs represent the early stage in an evolutionary sequence. Following the line of their work, we can think of $1422+202$ as a Medium-size Object having an asymmetric structure.

The core fractional luminosity is $\sim 4 \%$, a value consistent with the median value found for both CSSs and large size radio quasars. Relativistic boosting might be present in the core. However, only a weak and short jet coming out from the core is detected in the $\mathrm{VLA}_{15 \mathrm{GHz}}$ observation and it is not seen in the VLBI image, suggesting that the sub-arcsecond jet is not boosted. So, if we assume the average velocity of growth of $\sim 0.02 c$ for Medium-size Symmetric Object as in Fanti et al. (1995) the time required to grow for $1422+202$ will be $\geq 0.710^{7}$ years, larger than the CSSs age in the youth scenario.

The jet dominates the arcsecond scale structure of $1422+202$ at 5 and $8.4 \mathrm{GHz}$. The major axis Position Angle of the various components along the thin collimated jet indicates a possible helical shape. The origin of such a structure might be intrinsic to the core region or due to physical processes in the jet. Bends along the jet might be caused by interaction between the jet flow and dense gas 
clouds or could be the result of interference shocks in the plasma going down the jet (Hardee 1990). Such interactions can also brighten the emission. However, if the bends are formed by the former model we should see the effect of the interaction on the polarized emission. There is just a marginal indication of a difference in depolarization between the two sides of the source and the Faraday rotation of individual components is negligible going from $8.4 \mathrm{GHz}$ to $5 \mathrm{GHz}$ (Mantovani et al., in preparation). This lack also excludes frustration as an explanation for the asymmetric structure. The asymmetry is then the result of an intrinsic process.

The spectral index in $1422+220$ is everywhere steeper than 0.4. The core area has a spectrum which is flatter than the spectrum along the jet and at both the hot spot regions. The core shows a Giga-Hz-Peaked spectral index with the peak of emission at about $4 \mathrm{GHz}$. On the other hand, the resolution achieved by mapping the source with the VLA, did not allow to isolate the hot spots from the surrounding source structure. Their spectral indices are quite steep $(\alpha 1.0-1.6)$. Similar values were also found by Lonsdale \& Barthel (1984) for 3C 205 and by Carilli et al. (1992) for the high frequency part of the spectral index in both the hot spots of Cygnus A.

\subsection{Low frequency variability}

The variability reported for $1422+202$ at low frequency in monitoring observations (Bondi et al. 1994) has probably an origin extrinsic to the source. Refractive scintillation (Rickett 1986) can produce a variation of a few percent in flux density at frequencies $<1 \mathrm{GHz}$ in sources with compact bright structures similar to the hot spot found in $1422+202$. The source has a scintillation index of 0.018 (Bondi et al. 1994). Since the source is not point like, a compact component responsible for the refractive scintillation should be present inside its structure. Assuming a component which is $2-3 \mathrm{Jy}$ in flux density at $408 \mathrm{MHz}$ (the total mean flux of the source is $\sim 5 \mathrm{Jy}$ ), the scintillation index (rms flux density variation mean flux density ratio) will be $0.04-0.03$. The refractive scintillation theory (Rickett 1986) suggests that there is a correlation between the scintillation index, the galactic latitude and the source size. If we take a gaussian component as a simple case, from Fig. 4 of Spangler et al. (1993) we can derive a component size $\sim 30$ mas. The south hot spot has a component detected in our VLBI $18 \mathrm{~cm}$ observations which has a spectral index that extrapolated gives $2-3 \mathrm{Jy}$ of flux density at $408 \mathrm{MHz}$. This might explain the low frequency variability detected for $1422+202$ as due to an extrinsic mechanism, a point of view which is supported by the lack of flux density variation at frequencies $>2 \mathrm{GHz}$. However, this hot spot would have to have unusual properties among hot spots found in radio galaxies, which usually show angular sizes of the order of 200 mas. A VLBI obser- vation at low frequency is needed to confirm the existence of such a component.

\subsection{Phase referencing technique}

The successful application of the phase referencing technique to the source $1422+202$ allowed the detection of two components with absolute positions, relative to OQ 208, corresponding to the core region and to the south hot spot region of the radio source. This experiment was not designed as a pure astrometric one, with short duty cycle in switching between the reference and target source. Despite the fact that the reference source was observed for just three 13 minutes scans all along the 11 hours tracking of the target source, this experiment shows that the EVN can work as a phase stable instrument at $1.6 \mathrm{GHz}$ for separation up to $\sim 10^{\circ}$ between the sources and the reference calibrator.

The positional accuracy of $\sim 200$ mas is much worse than that usually achieved in astrometric experiments which is at $\mu$ arcsec level. However, the method can be extended to the cases where it is difficult to design a specific astrometric experiment.

Acknowledgements. F.M. thanks the Director, Onsala Space Observatory and L.B.B thanks the Director, Istituto di Radioastronomia, for their hospitality during periods when parts of the work presented here were done. We also like to thanks the correlator staff of the Max-Planck-Institut für Radioastronomie. The Onsala Space Observatory at the Chalmers University of Technology is the Swedish National Facility for Radioastronomy. The VLBI project at Onsala is supported by the Swedish National Science Foundation under grant F-FU 4876-302. Fredrik. T. Rantakyrö acknowledges support for his research by the European Union under contract ERBCHGECT920011. The National Radio Astronomy is operated by Associated Universities Incorporated under cooperative agreement with the National Science Foundation. AIPS is the NRAO's Astronomical Image Processing System.

\section{References}

Aoki S., Sôma M., Kinishita H., Inoue K., 1983, A\&A 128, 263 Argue A.N., Kenworthy C.N., Stewart P.M., 1973, ApJ 14, L99 Bååth L.B., 1991, in: Cornwell T.J. \& Perley R.A. (eds.) IAU Colloquium No. 131 Radio Interferometry Theory, Techniques and Applications, Astr. Soc. Pacif. Conf. Ser. 19, Astr. Soc. Pacif., San Francisco, p. 321

Bååth L.B., Mantovani F., 1991, in: Cornwell T.J. \& Perley R.A. (eds.) IAU Colloquium No. 131 Radio Interferometry Theory, Techniques and Applications, Astr. Soc. Pacif. Conf. Ser. 19, Astr. Soc. Pacif., San Francisco, p. 298

Bondi M., Padrielli L., Gregorini L., Mantovani F., Shapirovskaya N., Spangler S., 1994, A\&A 287, 390

Carilli C.L., Perley R.A., Dreher J.W., Leahy J.P., 1991, AJ 383,554

Fanti C., Fanti R., Dallacasa D., Schilizzi R.T, Spencer R.E, Stanghellini C., 1995, A\&A 302, 317

Hardee P.E., 1990, in: Parsec-scale radio jets, Zensus J.A., Pearson T.J. (eds.). Cambridge University Press, p. 282 
Mantovani F., Gregorini L., Padrielli L., Spangler S., 1990, Spangler S.R, Eastman W.A., Gregorini L., Mantovani F., A\&A 233, 535 Padrielli L., 1993, A\&A 267, 213

Mantovani F., Junor W., Fanti R., et al., 1992, MNRAS 257, Thompson A.R., Clark B.G., Wade C.M, Napier P.J., 1980, 353

Perley R.A., 1982, AJ 87, 859

Rickett B.J., 1986, AJ 307, 564 ApJS 44, 151

Véron-Cetty M.P., Véron P., 1987, ESO Scientific Report No. 5 